\title{
Análise da morbimortalidade dos pacientes com fraturas peritrocantéricas tratadas cirurgicamente com haste intramedular de fêmur proximal*
}

\section{Analysis of the Morbimortality of Patients with Peritrochanteric Fractures Surgically Treated with Proximal Femoral Intramedullary Rod}

\author{
Sidney Quintas ${ }^{1}$ Jacques Charlab ${ }^{1}$ Max Ramos $^{3}$ Henrique Mansur 2,30
}

1 Hospital Naval Marcílio Dias, Rio de Janeiro, RJ, Brasil
2 Hospital de Força Aérea de Brasília, Brasília, DF, Brasil
3 Universidade Federal do Estado do Rio de Janeiro (Unirio),
Rio de Janeiro, RJ, Brasil

Rev Bras Ortop 2019;54:396-401.

\author{
Endereço para correspondência Henrique Mansur, PhD Student \\ Hospital de Força Aérea de Brasília, Brasília, DF, Brasil \\ (e-mail: henrimansur@globo.com).
}

\begin{abstract}
Resumo
Palavras-chave

- fraturas do fêmur/ epidemiologia

- morbimortalidade

- fixação intramedular de fraturas

Objetivo Analisar a morbimortalidade dos pacientes com fraturas peritrocantéricas tratadas com haste intramedular e sua relação com o tempo de internação, com o tempo para fazer o procedimento cirúrgico, e com as comorbidades dos pacientes. Métodos Foi feito um estudo observacional, analítico e retrospectivo por meio da avaliação dos prontuários de 74 pacientes submetidos ao tratamento cirúrgico de fraturas peritrocantéricas com haste intramedular de fêmur proximal de 2011 a 2014 em uma unidade hospitalar.

Resultados A idade média no momento da ocorrência da fratura foi de 79,7 anos, e o tempo de internação total médio foi de 16,7 dias, com média de 11,3 dias até a cirurgia e 5,4 dias da cirurgia à alta. A incidência de complicações na internação no grupo com idade $\geq 78,5$ anos foi de $47,6 \%$, enquanto no grupo mais novo ela foi de $19,4 \%$ ( $p=0,013$ ). A incidência de complicações na internação no grupo que fez a cirurgia após 6 dias foi significativamente maior $(42,9 \% ; p=0,019)$. Observou-se também que a incidência de complicação na internação está significativamente associada ao risco cirúrgico de grau $\geq 3(p=0,001)$ e à diabetes mellitus $(p=0,001)$.

Conclusão As complicações relacionadas às fraturas peritrocantéricas estão significativamente associadas ao risco cirúrgico elevado (graus 3 e 4 ), diabetes mellitus, idade (> 78,5 anos) e tempo de internação pré-operatório prolongado (> 6 dias).
\end{abstract}

Trabalho feito no Hospital Naval Marcílio Dias, Rio de Janeiro, RJ, Brasil. Publicado originalmente por Elsevier Editora Ltda.

(D) Henrique Mansur's ORCID is https://orcid.org/0000-0001-7527969X.

recebido

18 de Dezembro de 2017

aceito

17 de Abril de 2018
DOI https://doi.org/

10.1016/j.rbo.2018.04.006. ISSN $0102-3616$.

\section{Introdução}

As fraturas do terço proximal do fêmur são causa de elevadas taxas de morbidade e mortalidade. ${ }^{1}$ Acometem principalmente os pacientes na faixa etária acima dos 50 anos, e as

Copyright $@ 2019$ by Sociedade Brasileira License terms de Ortopedia e Traumatologia. Published by Thieme Revnter Publicações Ltda, Rio de Janeiro, Brazil 


\section{Abstract}

\section{Keywords}

- femoral fractures/ epidemiology

- morbimortality

- intramedullary fracture fixation
Objective To analyze the morbimortality of patients with peritrochanteric fractures treated with intramedullary rod and its relationship to the length of hospital stay, the time to perform the surgical procedure, and the comorbidities of the patients.

Methods An observational, analytical and retrospective study was carried out through the evaluation of the medical records of 74 patients who underwent surgical treatment of peritrochanteric fractures with intramedullary proximal femoral rod from 2011 to 2014 in a hospital unit.

Results The mean age at the moment of the fracture was 79.7 years, and the mean total hospitalization time was 16.7 days, with an average of 11.3 days until surgery and 5.4 days from surgery to discharge. The incidence of complications during hospitalization in the group with aged $\geq 78.5$ years was of $47.6 \%$, while in the younger group it was of $19.4 \%(p=0.013)$. The incidence of hospitalization complications in the group that underwent surgery more than 6 days after the fracture was significantly higher: $42.9 \%(p=0.019)$. It was also observed that the incidence of complications during hospitalization was significantly associated with surgical risk index $\geq 3(p=0.001)$ and diabetes mellitus $(p=0.001)$.

Conclusion Complications related to peritrochanteric fractures are significantly related with high surgical risk index (grades 3 and 4), diabetes mellitus, age (> 78.5 years), and prolonged preoperative hospitalization ( $>6$ days). fraturas transtrocantéricas são mais incidentes em idosos a partir de 60 anos. $^{1}$

As fraturas que acometem a região proximal do fêmur podem ser divididas entre intracapsulares e extracapsulares. ${ }^{1}$ Essas fraturas têm grande associação com a osteoporose no paciente idoso, e são, na maioria dos casos, provenientes de traumas de baixa energia cinética, como quedas da própria altura. ${ }^{1,2} \mathrm{O}$ tratamento das fraturas do fêmur proximal é eminentemente cirúrgico, ${ }^{1,2}$ excetuando-se os casos nos quais o paciente não tem condições clínicas para fazer a operação. ${ }^{1-4} \mathrm{Na}$ análise da fratura peritrocantérica, deve-se identificar o grau de instabilidade que ela tem para que se faça a decisão correta quanto ao tratamento. ${ }^{5}$ Fatores como cominuição da parede posteromedial, traço de fratura reverso, e extensão subtrocantérica são considerados fatores de instabilidade dessas fraturas, e contribuem para uma redução mais trabalhosa e maior risco de falhas na síntese. ${ }^{1,5}$

Após a introdução dos dispositivos intramedulares com bloqueio cefálico, o tratamento desses padrões de fratura foi facilitado, ${ }^{1,5}$ e, aos poucos, tem substituído o parafuso deslizante de quadril (PDQ), que foi por muitos anos considerado o padrão-ouro para o tratamento das fraturas transtrocantéricas, especialmente no caso de fraturas instáveis. ${ }^{1}$ Os sistemas cefalomedulares são biomecanicamente mais favoráveis ${ }^{6}$ devido à redução do momento flexor, melhor controle rotacional, e pelo maior controle do colapso em varo e do encurtamento, decorrente da sua disposição mais medial, do que os dispositivos extramedulares. ${ }^{5}$

A fratura do fêmur proximal é considerada uma urgência ortopédica, e deve ser tratada entre 48 e 72 horas após o trauma, segundo a literatura. ${ }^{1} \mathrm{O}$ atraso no tratamento pode acarretar danos à saúde do paciente pelo longo período acamado, além de grande gasto de recursos por causa dessas complicações (úlcera de pressão, infecção do trato urinário [ITU], trombose venosa profunda e outras), ${ }^{7}$ que chegam a somar até 6 bilhões de dólares apenas com gastos clínicos nos Estados Unidos. ${ }^{8}$

Este trabalho tem como objetivo avaliar a morbimortalidade dos pacientes com fratura peritrocantérica tratados cirurgicamente com haste intramedular de fêmur proximal, e sua relação com o tempo de internação, com o tempo para fazer o procedimento cirúrgico, e com as comorbidades dos pacientes.

\section{Material e Métodos}

Este estudo contou com 74 pacientes, e foi caracterizado como observacional, retrospectivo e analítico. Em um hospital militar, fez-se um levantamento de dados dos prontuários dos pacientes submetidos ao tratamento cirúrgico de fraturas peritrocantéricas (transtrocantéricas ou subtrocantéricas) com haste intramedular de fêmur proximal, de 2011 a 2014, após aprovação do CEP da instituição, com registro na Plataforma Brasil sob o número do CAAE: 71991417.4.0000.5256.

Foram excluídos: os pacientes não submetidos ao tratamento cirúrgico; os esqueleticamente imaturos; e os politraumatizados ou que apresentavam mais de uma fratura que necessitasse de cirurgia na mesma internação. Por fim, foram excluídos os pacientes com prontuários incompletos ou que tiveram acompanhamento pós-operatório menor do que seis meses, exceto em casos de óbito nesse período.

A partir dos dados coletados, elaborou-se um banco de dados em planilha eletrônica para analisá-los com os programa Statistical Package for the Social Sciences (SPSS, IBM Corp., Armonk, NY, EUA), versão 22.0, e Microsoft Excel 2007 (Microsoft, Redmond, WA, EUA). 
Para a identificação de um ponto de corte ótimo para variáveis associadas com as complicações, que maximizasse o risco de complicação, usou-se a metodologia da curva característica de operação do receptor (COR), e, para investigar a associação significativa entre duas variáveis qualitativas, usou-se o teste qui-quadrado ou, quando o qui-quadrado se mostrou inconclusivo e a situação foi adequada, o teste exato de Fisher. Todas as discussões consideraram nível de significância máximo de $5 \%(p \leq 0,05) .{ }^{9,10}$

\section{Resultados}

A amostra base desta pesquisa foi formada por 74 pacientes; 7 deles não tinham informação de sexo, 50 eram do sexo feminino, e 17, do masculino. Portanto, houve predomínio significativo de mulheres $(74,6 \%)$ na população de pacientes submetidos à cirurgia para correção das fraturas peritrocantéricas $(p=0,000)$. Após a análise dos dados coletados, foi observada uma idade média no momento da ocorrência da fratura de 79,7 anos, e um tempo de internação total médio de 16,7 dias, com uma média de 11,3 dias até a cirurgia, e de 5,4 dias da cirurgia à alta.

Complicações durante a internação ocorreram em 27 casos (36,5\%), e foram registrados 11 casos que evoluíram com alguma complicação no período pós-internação (14,9\%). Houve registro de 14 óbitos: 2 (2,7\%) em nova internação pós-operatória, 4 nos 2 primeiros meses pós-cirurgia, 1 aos 4 meses após a cirurgia, 1 aos 10 meses pós-cirurgia, 1 aos 12 meses após a cirurgia, e 5 depois de mais de 12 meses após a cirurgia. A distribuição das incidências de interesse pode ser vista na - Figura 1.

Os pacientes estudados apresentaram uma diversidade de intercorrências durante a internação. As complicações mais prevalentes durante a internação foram infecção do trato urinário (ITU) em $21(28,4 \%)$ pacientes, dispneia tratada e não complicada em $4(5,4 \%)$ casos, pneumonia em $3(4,1 \%)$ casos, delirium em $2(2,7 \%)$ pacientes, e fibrilação atrial em 2 $(2,7 \%)$ pacientes. Todas as outras intercorrências foram casos isolados, e corresponderam, cada uma, a 1,4\%. Foram elas: descompensação cardíaca e respiratória, anemia, dor retroesternal, e crise hipertensiva. A - Tabela 1 traz a incidências de complicações na internação e de complicações pós-operatórias em subgrupos de interesse.
As complicações na internação não estiveram associadas ao sexo do paciente $(p=0,842)$, nem ao lado $(p=0,753)$ ou tipo de fratura $(p=0,516)$. As complicações pós-cirúrgicas não estiveram associadas a quaisquer das comorbidades analisadas e nem ao tipo de fratura $(p=1,000)$. Entretanto, a incidência de complicação na internação esteve significativamente associada ao risco cirúrgico $(\mathrm{RC})$ de grau $\geq 3$ ( $p=$ $0,001)$ e ao diabetes mellitus (DM) $(p=0,001)$.

A incidência de complicações entre os pacientes com RC de grau 1 ou 2 foi de 15,2\%; e entre os que tinham RC 3 ou 4, foi significativamente maior: $52,5 \%$. Estima-se que a chance de um paciente com fratura peritrocantérica com RC 3 ou 4 ter complicação na internação é 6,2 vezes maior do que a chance de um paciente com fratura com RC 1 ou 2 .. A incidência de complicações entre os pacientes que não tinham DM foi de $24,0 \%$; e entre os que tinham DM foi significativamente maior: $62,5 \%$. Estima-se que a chance de um paciente com fratura peritrocantérica portador de DM ter complicação na internação é 5,3 vezes maior do que a chance de um paciente com fratura peritrocantérica não portador de DM.

A - Tabela 2 traz as principais estatísticas de idade, tempo total de internação, tempo da internação até a cirurgia, e tempo da cirurgia a alta, dos pacientes que tiveram e não tiveram complicações na internação. Os valores de $p$ foram todos menores do que 0,05 ; isso mostra que a complicação na internação esteve significativamente associada à idade do paciente e aos tempos de internação. Houve diferença significativa entre as distribuições do tempo de internação total dos pacientes que não tiveram complicações pós-cirúrgicas e dos pacientes que tiveram complicações pós-cirúrgicas, bem como no tempo entre a internação e a cirurgia, e no tempo da cirurgia à alta hospitalar, ambos com uma diferença significativa nos pacientes que tiveram ou não complicações póscirúrgicas. A complicação pós-cirúrgica não esteve associada de forma significativa à idade do paciente ou aos tempos de internação.

Na busca de uma idade de corte, fez-se uma análise por curva COR que identificou o valor de 78,5 como ponto de corte da idade que maximiza o risco para a complicação na internação.

A - Tabela 3 traz a análise da incidência de complicações nos pacientes com menos de 78,5 anos e mais de 78,5 anos. $\mathrm{A}$

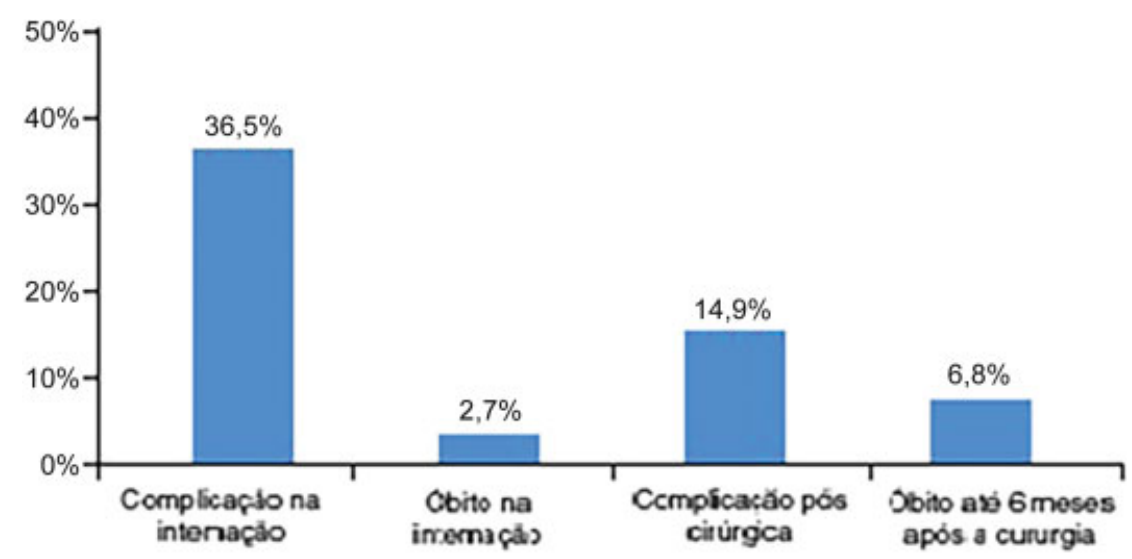

Fig. 1 Incidências de complicações e óbitos dos pacientes submetidos à cirurgia para correção das fraturas peritrocantéricas. 
Tabela 1 Incidências de complicações pós-cirúrgicas e na internação em subgrupos de interesse

\begin{tabular}{|c|c|c|c|c|c|c|}
\hline \multirow[t]{2}{*}{ Subgrupo } & \multicolumn{2}{|c|}{$\begin{array}{l}\text { Incidência de } \\
\text { complicações } \\
\text { na internação }\end{array}$} & \multirow[t]{2}{*}{ Valor de $p$} & \multicolumn{2}{|c|}{$\begin{array}{l}\text { Incidência de } \\
\text { complicações } \\
\text { pós-cirúrgicas }\end{array}$} & \multirow[t]{2}{*}{ Valor de $p$} \\
\hline & $n$ & $\%$ & & $\mathrm{n}$ & $\%$ & \\
\hline \multicolumn{7}{|l|}{ Sexo } \\
\hline Feminino & 19 & $38,0 \%$ & 0,842 & 6 & $12,0 \%$ & 1,000 \\
\hline Masculino & 2 & $11,8 \%$ & & 2 & $11,8 \%$ & \\
\hline \multicolumn{7}{|c|}{ Risco cirúrgico (graus) } \\
\hline 1 ou 2 & 5 & $15,2 \%$ & 0,001 & 5 & $15,2 \%$ & 1,000 \\
\hline 3 ou 4 & 21 & $52,5 \%$ & & 6 & $15,0 \%$ & \\
\hline \multicolumn{7}{|l|}{ HAS } \\
\hline Sem HAS & 4 & $23,5 \%$ & 0,206 & 4 & $23,5 \%$ & 0,263 \\
\hline Com HAS & 23 & $40,4 \%$ & & 7 & $12,3 \%$ & \\
\hline \multicolumn{7}{|l|}{$D M$} \\
\hline Sem DM & 12 & $24,0 \%$ & 0,001 & 6 & $12,0 \%$ & 0,321 \\
\hline Com DM & 15 & $62,5 \%$ & & 5 & $20,8 \%$ & \\
\hline \multicolumn{7}{|l|}{ Tipo de fratura } \\
\hline Subtrocantérica & 3 & $27,3 \%$ & 0,516 & 2 & $18,2 \%$ & 1,000 \\
\hline Transtrocantérica & 20 & $39,2 \%$ & & 8 & $15,7 \%$ & \\
\hline
\end{tabular}

Abreviaturas: DM, diabetes mellitus; HAS, hipertensão arterial sistêmica.

Tabela 2 Principais estatísticas de idade, tempo total de internação, tempo da internação até a cirurgia, e tempo da cirurgia à alta dos pacientes que tiveram e não tiveram complicações na internação

\begin{tabular}{|l|l|l|l|l|l|l|l|l|}
\hline & \multicolumn{2}{|l|}{ Estatística } & \multicolumn{2}{l|}{$\begin{array}{l}\text { Tempo de internação } \\
\text { total }\end{array}$} & \multicolumn{2}{l|}{$\begin{array}{l}\text { Tempo de internação } \\
\text { até a cirurgia }\end{array}$} & \multicolumn{2}{l|}{$\begin{array}{l}\text { Tempo da cirurgia } \\
\text { até a alta hospitalar }\end{array}$} \\
\hline & $\begin{array}{l}\text { Sem } \\
\text { complicação }\end{array}$ & $\begin{array}{l}\text { Com } \\
\text { complicação }\end{array}$ & $\begin{array}{l}\text { Sem } \\
\text { complicação }\end{array}$ & $\begin{array}{l}\text { Com } \\
\text { complicação }\end{array}$ & $\begin{array}{l}\text { Sem } \\
\text { complicação }\end{array}$ & $\begin{array}{l}\text { Com } \\
\text { complicação }\end{array}$ & $\begin{array}{l}\text { Sem } \\
\text { complicação }\end{array}$ & $\begin{array}{l}\text { Com } \\
\text { complicação }\end{array}$ \\
\hline Mínimo & 42 & 68 & 6 & 9 & 3 & 4 & 2 & 2 \\
\hline Máximo & 96 & 97 & 30 & 64 & 20 & 54 & 13 & 31 \\
\hline Média & 77,4 & 83,2 & 13,7 & 22,7 & 9,6 & 14,9 & 4,0 & 7,7 \\
\hline Mediana & 78 & 83 & 13 & 18 & 9 & 12 & 4 & 5 \\
\hline $\begin{array}{l}\text { Desvio } \\
\text { padrão }\end{array}$ & 10,2 & 6,6 & 5,4 & 14,0 & 4,4 & 10,7 & 1,7 & 7,5 \\
\hline $\begin{array}{l}\text { Coeficiente } \\
\text { de variação }\end{array}$ & 0,13 & 0,08 & 0,39 & 0,62 & 0,45 & 0,72 & 0,42 & 0,97 \\
\hline $\begin{array}{l}\text { Valor de } p \\
\text { do teste de } \\
\text { Mann-Whitney }\end{array}$ & 0,001 & & 0,010 & & 0,003 & & & 0,010 \\
\hline
\end{tabular}

Tabela 3 Incidências de complicações dos pacientes submetidos à cirurgia para correção das fraturas peritrocantéricas

\begin{tabular}{|l|l|l|l|l|l|l|l|}
\hline $\begin{array}{l}\text { Incidência de } \\
\text { complicações }\end{array}$ & \multicolumn{2}{l|l|l|l|}{$\begin{array}{l}\text { Idade }<\mathbf{7 8 , 5} \text { anos } \\
(\mathbf{n}=\mathbf{3 1})(\%)\end{array}$} & \multicolumn{2}{l|}{$\begin{array}{l}\text { Idade }>\mathbf{7 8 , 5} \text { anos } \\
(\mathbf{n}=\mathbf{4 2})(\%)\end{array}$} & $\begin{array}{l}\text { Valor de } \boldsymbol{p} \text { do teste } \\
\text { do qui-quadrado }\end{array}$ & $\begin{array}{l}\text { RP } \\
\text { IC95\% RP }\end{array}$ \\
\hline Na internação & 6 & $(19,4 \%)$ & 20 & $(47,6 \%)$ & 0,013 & 3,8 & $1,3-11,1$ \\
\hline Pós-cirúrgicas & 5 & $(16,1 \%)$ & 6 & $(14,3 \%)$ & 1,000 & 0,87 & $0,2-3,2$ \\
\hline
\end{tabular}

Abreviaturas: IC95\%, intervalo de confiança de 95\%; RP, razão de possibilidades.

incidência de complicação na internação no grupo com idade $<78,5$ anos foi de $19,4 \%$; já a incidência de complicações na internação no grupo com idade $\geq 78,5$ anos foi significativamente maior: $47,6 \%(p=0,013)$.

$\mathrm{Na}$ busca de um ponto de corte para o tempo de internação até a cirurgia, fez-se uma análise por curva COR que identificou o valor de 6,5 como ponto de corte do tempo de internação que maximiza o risco para a complicação na internação.

A - Tabela 4 faz uma comparação das incidências de complicações nos pacientes com tempo de internação e cirurgia até 6,5 dias e de mais de 6,5 dias. A incidência de complicação na internação no grupo que fez cirurgia após 6 
Tabela 4 Incidências de complicações dos pacientes submetidos à cirurgia para correção das fraturas peritrocantéricas

\begin{tabular}{|l|l|l|l|l|l|}
\hline $\begin{array}{l}\text { Incidência de } \\
\text { complicações }\end{array}$ & $\begin{array}{l}\text { Cirurgia em até } \\
\mathbf{6} \text { dias }(\mathbf{n}=\mathbf{1 7})(\%)\end{array}$ & $\begin{array}{l}\text { Cirurgia após 6 dias de } \\
\text { internação }(\mathbf{n}=\mathbf{5 6})(\%)\end{array}$ & Valor de $\boldsymbol{p}$ & RP & 95\%IC da RP \\
\hline Na internação & $2(11,8 \%)$ & $24(42,9 \%)$ & 0,019 & 5,6 & $1,2-27,0$ \\
\hline Pós-cirúrgicas & $1(5,9 \%)$ & $10(17,9 \%)$ & 0,439 & 3,5 & $0,4-29,4$ \\
\hline
\end{tabular}

Abreviaturas: IC95\%, intervalo de confiança de 95\%; RP, razão de possibilidades.

dias de internação foi significativamente maior $(p=0,019)$. Estima-se então que a chance de um paciente que leva mais de 6 dias para ser operado ter alguma complicação na internação é 5,6 vezes maior do que a chance de um paciente que foi operado em até 6 dias.

\section{Discussão}

As fraturas peritrocantéricas estão relacionadas a índices elevados de morbidade e mortalidade. ${ }^{1-4} \mathrm{~A}$ incidência geral de mortalidade após correção cirúrgica das fraturas trocantéricas é descrita na literatura atual entre $6 \%$ e $11 \%$ no primeiro mês, e entre $14 \%$ e $36 \%$ no primeiro ano. ${ }^{11}$ Neste estudo, verificou-se um índice de mortalidade de 18,9\%; 6,8\% dos óbitos aconteceram dentro dos 6 primeiros meses de pósoperatório.

Alguns artigos desconsideraram a relação do tipo de fratura, idade e número de comorbidades com o risco de mortalidade, ${ }^{12}$ enquanto outros autores fizeram levantamentos com relevância estatística e estreitaram a relação da idade ( $>80$ anos) e do número de comorbidades (maior do que 2) com uma taxa maior de mortalidade. ${ }^{3,4}$ Ainda são encontradas taxas conflitantes na literatura em relação à mortalidade e ao tempo até a cirurgia, as quais variam entre uma relação íntima da menor mortalidade dos pacientes tratados até 72 horas depois da fratura, ${ }^{1,2,13}$ e nenhuma diferença na mortalidade, mesmo com o atraso do procedimento cirúrgico. ${ }^{8,14,15}$

Nossa análise da mortalidade do pós-operatório não indicou relevância estatística por causa de valores pequenos e isolados que não poderiam ser relacionados com a cirurgia diretamente, mas a morbidade (complicações) durante a internação nos pacientes operados após 6 dias de fratura se mostrou significativamente maior $(42,9 \%)$ em relação à dos operados em até 6 dias $(11,8 \%)(p=0,019)$. As complicações pós-operatórias também foram mais elevadas naqueles operados com mais de 6 dias de internação (17,9\%) em relação aos que operaram em até 6 dias (5,9\%), porém com menor diferença quando comparadas com as complicações durante a internação $(p=0,439)$. Observou-se que os pacientes operados após 6 dias da fratura têm 5,6 vezes mais chances de ter uma complicação durante a internação em relação àqueles que operam em menos de 6 dias.

O sexo feminino foi consideravelmente mais acometido $(74,6 \%)$ pelas fraturas peritrocantéricas nos dados coletados no presente estudo, o que corresponde aos achados da literatura (74-80\%), ${ }^{1-4,12}$ além de ter sido o sexo que apresentou mais complicações durante a internação $(p=0,842)$. Embora os valores encontrados nesta análise mostrem um índice de complicações pós-operatórias semelhante em ambos os sexos $(p=1,000)$, há na literatura evidências de que o sexo masculino é expressivamente mais suscetível a complicações pós-operatórias, assim como tem maior mortalidade em até um ano de pós-operatório. ${ }^{16,17}$ Neste artigo, o sexo do paciente não se mostrou estatisticamente relevante no tocante às complicações $(p=0,842)$.

Diversos autores fizeram análises de mortalidade e morbidade do pós-operatório em relação ao tempo de internação, à idade e às comorbidades; ${ }^{1,2,8,11-14,17-19}$ porém, faltam estudos no que diz respeito às complicações durante a internação. Um estudo que avaliou os efeitos no atraso do tratamento das fraturas do fêmur proximal verificou que as complicações intra-hospitalares ocorreram mais frequentemente $(56,1 \%$ dos casos) nos pacientes que tiveram atraso de 7 dias para fazer a cirurgia; o prolongamento da internação pós-operatória imediata e das complicações pós-operatórias também foram mais frequentes nesse mesmo grupo. ${ }^{7} 0$ índice de complicações para o grupo que operou em 48 horas foi de $16,5 \%{ }^{7}$ Esse resultado se assemelha bastante ao encontrado no presente trabalho, em que mais de 6 dias de hospitalização antes da cirurgia aumentaram significativamente o risco de complicações.

As complicações mais frequentes na internação encontradas na literatura são úlceras de pressão (17,4\%), ITU (17\%), e trombose venosa profunda $(9,4 \%) .^{7}$ As complicações mais frequentes observadas no presente estudo foram ITU $(28,4 \%)$, dispneia (5,4\%), e pneumonia $(4,1 \%)$. Outros fatores, como o RC de grau 3 ou 4, idade $>78$ anos, e a presença de DM como comorbidade, também têm grande efeito sobre o risco de haver complicações na internação.

A idade acima de 78,5 anos constituiu um fator de risco aumentado e significativo para o desenvolvimento de complicações na internação ( $p=0,013)$; porém, a mesma coisa não foi observada pós-operatoriamente, após a alta hospitalar. As referências reunidas citam a faixa etária entre 70 e 80 anos como fator importante para complicações pós-operatórias, porém não são específicas quanto às complicações pré-cirúrgicas ou durante a internação. ${ }^{13-17,19,20}$

A literatura também apresenta evidências compatíveis com os achados deste trabalho quando analisa a correlação do $\mathrm{RC}$ e das comorbidades como fatores predisponentes a complicações. As complicações mais graves da internação citadas foram infecção pulmonar e parada cardíaca (não especificada), que se mostraram diretamente ligadas ao número de comorbidades (três ou mais) e ao RC com grau mais elevado. A correlação direta da DM com as complicações intra e extra-hospitalares não é clara nas referências; o número de comorbidades é o principal fator citado. ${ }^{14,15,17,19}$ 
Acreditamos que a limitação do número da amostra pode ter diminuído a significância estatística de alguns fatores estudados. Porém, nosso estudo ratifica a importância da maior atenção e agilidade na assistência às fraturas peritrocantéricas com indicação de tratamento cirúrgico com o uso de implantes como a haste intramedular. Apesar de não termos observado um grande número de complicações e intercorrências no pósoperatório, estas estão diretamente relacionadas ao gasto excessivo com patologias clínicas e ao aumento da morbidade nos pacientes com tempo de internação pré-operatório prolongado. Além disso, nos pacientes idosos e osteoporóticos, que, consequentemente, apresentam risco maior de sofrerem fraturas peritrocantéricas do fêmur, é importante o controle adequado das comorbidades, especialmente a DM, por conta da taxa maior de complicações.

\section{Conclusão}

As fraturas peritrocantéricas tratadas com haste intramedular proximal de fêmur apresentaram mortalidade de 12,6\% no primeiro ano pós-operatório, e morbidade de 51,4\%. A complicação na internação está significativamente associada à idade do paciente, ao tempo de internação, ao RC de grau 3 ou 4, e à presença de DM como comorbidade associada. Entretanto, as complicações após a alta hospitalar não apresentam correlação significativa com o tempo entre a internação e a cirurgia e a idade dos pacientes.

Conflitos de Interesse

Os autores declaram não haver conflitos de interesse.

\section{Referências}

1 Bucholz RW, Heckman JD, Court-Brown CM, Tornetta P III, McQueen MM, Ricci WM. Fraturas em adultos de Rockwood e Green. 7th ed. Barueri: Manole; 2013

2 Canale T, Beaty J. Campbell's Opertive Orthopaedics. 12th ed. Philadelphia: Elsevier; 2013

3 Hebert SK, Barros Filho TEP, Xavier R, Pardini Júnior AG. Ortopedia e Traumatologia: Princípios e Prática. 4th ed. Porto Alegre: Art Med; 2009

4 Barros Filho TEP, Camargo OP, Camanho GL. Clínica Ortopédica. Barueri: Manole; 2012
5 Baumgaertner MR, Curtin SL, Lindskog DM. Intramedullary versus extramedullary fixation for the treatment of intertrochanteric hip fractures. Clin Orthop Relat Res 1998;(348):87-94

6 Rüedi TP. Princípios AO do tratamento de fraturas. 2nd ed. Porto Alegre: Art Med; 2009

7 Rodriguez-Fernandez P, Adarraga-Cansino D, Carpintero P. Effects of delayed hip fracture surgery on mortality and morbidity in elderly patients. Clin Orthop Relat Res 2011;469(11):3218-3221

8 Sakaki MH, Oliveira Rocha A, Coelho FF, Leme Garcez LE, Suzuki I, Amatuzzi MM. Estudo da mortalidade na fratura do fêmur proximal em idosos. Acta Ortop Bras 2004;12(04):242-249

9 Medronho RA, Bloch KV, Luiz RR, Werneck GL. Epidemiologia. São Paulo: Atheneu; 2009

10 Pagano M, Gauvreau K. Princípios de Bioestatística. São Paulo: Pioneira Thomson Learning; 2004

11 Borger RA, Leite FA, Araújo RP, Pereira TFN, Queiroz RD. Avaliação prospectiva da evolução clínica, radiográfica e funcional do tratamento das fraturas trocantéricas instáveis do fêmur com haste cefalomedular. Rev Bras Ortop 2011;46(04):380-389

12 Daniachi D, Netto AdosS, Ono NK, Guimarães RP, Polesello GC, Honda EK. Epidemiologia das fraturas do terço proximal do fêmur em pacientes idosos. Rev Bras Ortop 2015;50(04):371-377

13 Shiga T, Wajima Z, Ohe Y. Is operative delay associated with increased mortality of hip fracture patients? Systematic review, meta-analysis, and meta-regression. Can J Anaesth 2008;55(03):146-154

14 Grimes JP, Gregory PM, Noveck H, Butler MS, Carson JL. The effects of time-to-surgery on mortality and morbidity in patients following hip fracture. Am J Med 2002;112(09):702-709

15 Stoddart J, Horne G, Devane P. Influence of preoperative medical status and delay to surgery on death following a hip fracture. ANZ J Surg 2002;72(06):405-407

16 Mesquita GV, Lima MAL, Santos AMR, Alves ELM, Brito JNP, Martins MCÇ. Morbimortalidade em idosos por fratura proximal do fêmur. Texto Contexto Enferm Florianópolis 2009;18(01):67-73

17 Roche JJ, Wenn RT, Sahota O, Moran CG. Effect of comorbidities and postoperative complications on mortality after hip fracture in elderly people: prospective observational cohort study. BMJ 2005;331(7529):1374

18 Kannegaard PN, van der Mark S, Eiken P, Abrahamsen B. Excess mortality in men compared with women following a hip fracture. National analysis of comedications, comorbidity and survival. Age Ageing 2010;39(02):203-209

19 Lawrence VA, Hilsenbeck SG, Noveck H, Poses RM, Carson JL. Medical complications and outcomes after hip fracture repair. Arch Intern Med 2002;162(18):2053-2057

20 Lefaivre KA, Macadam SA, Davidson DJ, Gandhi R, Chan H, Broekhuyse HM. Length of stay, mortality, morbidity and delay to surgery in hip fractures. J Bone Joint Surg Br 2009;91(07):922-927 